Z. klin. Chem. u. klin. Biochem.

7. Jg., S. 617-623, November 1969

\title{
Veränderungen der Gc-Varianten durch enzymatische Einwirkungen ${ }^{1}$ )
}

\author{
Von R. HilgermanN \\ Aus dem Institut für gerichtliche Medizin der Universität Marburg (Direktor: Prof. Dr. F. Scbleyer)
}

(Eingegangen am 30. Juni 1969)

Die intrazellulären Proteasen (Kathepsine) bewirken nach ihrer Freisetzung (Hämolyse) deutliche immunoelektrophoretisch darstellbare Transformationen der Gc-Globuline und einiger anderer Serumproteine. Eine Inaktivierung dieser Kathepsine durch bekannte polyvalente Proteasen-Inhibitoren war nicht möglich. Hinsichtlich ihrer Proteinsubstratspezifität besteht keine biochemische Identität zwischen den Kathepsinen und den proteolytischen Verdauungsenzymen (Endopeptidasen).

\section{Alterations of the Gc-variants by encyme action}

The intracellular proteases (cathepsins) induce distinct immunoelectrophotetic transformations of the group-specific component (Gc) and other serum proteins after their liberation by haemolysis. These cathepsins could not be inactivated by polyvalent protease inhibitors. With regard to their protein substrate specifity, there is no biochemical identity between the cathepsins and the proteinsplitting enzymes (endopeptidases) secreted into the gastrointestinal tract.

SCHULTZE und ScHwick (1) haben untersucht, in welchem Maße chemisch-physikalische, fermentative und Einwirkungen durch Krankheiten das Bild der Immunoelektrophorese zu beeinflussen vermögen. Unter diesen Faktoren führt die Einwirkung proteolytischer Fermente zu den stärksten Veränderungen in der Proteinstruktur: Dislokation, Doppelkonturierung, Aufteilung, Abschwächung oder sogar Verschwinden typischer Immunpräzipitate. LEITHOFF und LEITHOFF (2) stellten bei der immunoelektrophoretischen Differenzierung der Proteine faulen Leichenblutes eine gewisse Rangordnung des Schwundes der Proteinfraktionen auf, die auch von anderen Autoren bestätigt wurde $(3,4)$. Danach ist, von einigen Ausnahmen abgesehen, die Stabilität gegenüber Fäulnis und Autolyse eine Funktion der Quantität: die quantitativ stätksten Proteinfraktionen widerstehen fermentativen Einflüssen am längsten.

Hieraus ist abzuleiten, da $\beta$ die quantitativ geringeren, niedermolekularen und sich in sehr feinen Präzipitatlinien abzeichnenden Gc-Proteine naturgemäß früher und schwerer geschädigt werden (3). $\mathrm{Daß}$ die Wirkung proteolytischer Eigenfermente der Blutzellen und zahlreicher Bakterienfermente die Ursache der immunoelektrophoretisch nachweisbaren Veränderungen der gruppenspezifischen Komponente ist, wurde von NERSTRøM $(5,6)$ und Nerstrøm und Mitarbeitern $(7,8)$ durch systematische Untersuchungen bewiesen. Im Vordergrund stehen auch hier Unregelmäßigkeiten der Wanderung der Gc-Präzipitate und ihrer sog. Leitpräzipitate oder Bezugsbögen. Bei fortgeschrittener „Transforma-

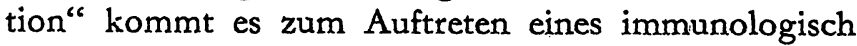
mit der gruppenspezifischen Komponente identischen $\alpha_{1}$-Globulins.

Inzwischen liegen weitere Kenntnisse über Transformationen der Gc-Präzipitate u. a. nach thermischen Einflüssen, an Leichenblut, Blutspuren und an gelagertem Blut in Abhängigkeit vom Zeitfaktor vor $(3,4,9,10,11,12,13)$.

1) Als Kurzvortrag gehalten auf der Tagung der Gesellschaft für forenische Blutgruppenkunde, Travemünde 1969.
Die Transformation der Gc-Typen und der Serumproteine durch freigesetzte proteolytische Fermente aus desintegrierten Leukocyten und Thrombocyten gilt wohl als unumstritten. Umstritten ist aber die Frage, welcbe intrazellulären Enzyme für diese Proteolyse verantwortlich sind.

Auf der Suche nach den am katabolen intrazellulären Proteinstoffwechsel beteiligten Hydrolasen wurden bisher in tierischen und zum Teil auch in menschlichen Geweben die Kathepsine $A$ bis $E$ beschrieben (14). Für die Kathepsine $A$ bis $D$ konnte ihre lysosomale Lokalisation nachgewiesen werden $(15,16,17$, 18 u. a.). Als Lysosomen bezeichnet man eine Gruppe von zytoplasmatischen Teilchen, die bei der üblichen Zellfraktionierung als eine Mittelfraktion zwischen Mitochondrien und Mikrosomen auftritt. Kathepsin ist eine Sammelbezeichnung für die Vielzahl der proteolytischen Enzyme in den Gevebszellen mit verschiedenen pH-Optima und Spezifitäten. Nach ihrer Spezifität gegenüber synthetischen Substraten bestehen gewisse Ähnlichkeiten des Kathepsins A mit dem Pepsin, des Kathepsins B mit dem Trypsin und des Kathepsins $\mathrm{C}$ mit dem Chymotrypsin. Die proteolytische Hauptaktivität dürfte den Kathepsinen der Gruppe D zukommen, für die bisher keine synthetischen Substrate gefunden wurden, und die von Fruton (19) als "hämoglobinspaltende“ Kathepsine bezeichnet wurden (14). $\mathrm{Da}$ es bisher nicht gelungen ist, diese zellulären Enzyme in kristalliner Form rein darzustellen, bestehen noch viele Unklarheiten hinsichtlich Identität und spezifischer Eigenschaften der einzelnen Kathepsine.

Ähnlich unbefriedigend sind aus den gleichen Gründen die bisherigen Erkenntnisse über die proteolytischen Enzyme der Blutzellen. Nach Panturtschiko und Stattarann (20) kommt eine Protease mit eincm pH-Optimum bei 7,4 in den Lethkocyten vor, die nach ihrem Verhalten gegen verschiedene Substrate nicht mit Trypsin identisch ist, wie früher angenommen wurde. Außerdem werden ein Kathepsin mit einem pH-Optimum von 4,5 und Peptidasen in den Zellen der myeloischen und der lymphatischen Reihe beschrieben. HASCHEN und Mitarbeitcr $(21,22)$ fanden in Ubereinstimmung mit Frer und Mitarbeitern (23) eine neutrale Protease (pH 7,5) in den Leukocyten; ferner beschrieb HASCHEN $(21,22)$ noch eine saure Protease ( $\mathrm{pH} 3,5)$ und 7 Peptidasen einschließlich einer katheptischen Carboxypeptidase. Mounrer und ATrYer (24) haben 3 katheptische Enzymaktivitäten gemessen, eine in Leukocyten mit einem pH-Optimum ron 8,0 , die dem Chymotrypsin ähneln soll, und 2 weitere aus Lymphocyten mit einem pH-Optimum von 3,0 und 5,5. STILES und FraENKELConRat (25) beschrieben glcichfalls zwei pH-Optima bei 3,5 und 
8,5; sie wiesen ein Überwiegen der sauren Protease bei den Lymphocyten und der alkalischen Protease bei den Leukocyten nach. Ferner konnten sie zeigen, daß die proteolytischen Aktivitäten, wenn auch in unterschiedlicher Verteilung, praktisch in allen Zellfraktionen vorhanden sind, also nicht nur in den neutrophilen Granula der Leukocyten, die nach Aufbau und Enzymgehalt als Lysosomen anzusehen sind (26).

Die menschlichen Thrombocyten enthalten ein Kathepsin A, eine Protease-Aktivität bei $\mathrm{pH}$ 3,5 und sechs weitcre Exopeptidasen, die hinsichtlich pH-Optimum, nicht aber quantitativ mit den Erythrocyten-Enzymen übereinstimmen (27, 28). Auf das Zellrolumen bezogen, übertrifft ihre Aktivität sogar diejenige intraleukocytärer Fermente (29).

Bemerkenswert ist schließlich, daß auch die Erythrog'ten neben zahlreichen Peptidasen eine Protease-Aktivität mit einem $\mathrm{pH}-$ Optimum bei 7,4 aufweisen und eine saure Protease mit einem pH-Optimum bei 3,0, die in ihrem Verhalten dem Kathepsin D nahesteht $(30,31,32)$.

Nerstrøin (6) hat nachgewiesen, daß die Gc-Komponenten durch die Proteasen aus Leukocyten und Thrombocyten getrennt voneinander in ähnlicher Weise transformiert werden. Es ergab sich nun die praxisnahe Fragestellung, ob sich die intrazellulären Proteasen mit einem der bekannten polyvalenten Protease-Inhibitoren inaktivieren lassen, und so das Auftreten von Gc-Defekttypen vermeidbar ist. Gleichzeitig war zu prüfen, ob sich bei einer spezifischen Hemmung dieser Zellfermente und durch den Vergleich transformierter Gc-Typen mit den Präzipitatmustern, die nach Einwirken bekannter Verdaunngsfermente entstehen, gewisse Übereinstimmungen im proteolytischen Verhalten der Fermentsysteme der Blutzellen und des Verdaunngstraktes nachweisen lassen; denn diese Identität ist biochemisch noch keineswegs geklärt.

\section{Material und Methoden}

\section{Herstellung der Substrate}

Leukocyten und Thrombocyten wurden aus den bei der Plasmaherstellung gewonnenen Blutzellen frischer Blutkonserven isoliert ${ }^{2}$ ). Die Zellen wurden in 0,85 proz. $\mathrm{NaCl}$-Lösung suspendiert und $3 \mathrm{mal}$ bei $3000 \mathrm{U} . / \mathrm{Min}$. zentrifugiert. Dabei wurden jeweils die obersten erythrocytenfreien Zellschichten sorgfältig abgehoben, anschließend unter Kühlung mit einem Ultra-Turrax (Janke \& Kunkel, 7813 Staufen) homogenisiert und schließlich bei $-20^{\circ}$ aufbewahrt. Eine weitere Trennung dex Leukocytcn von Lymphocyten und Thrombocyten wurde nicht vorgenommen.

Erythrocyten wurden aus frisch entnommenem Citratblut nach dem von BETKE (33) angegebenen Verfahren isoliert, gleichfalls homogenisiert und bei $-20^{\circ}$ eingefroren. Die Erythrocytensuspension wurde außerdem im Blutausstrich sowie in der Zählkammer nach Zusatz von HAYEMscher Lösung mikroskopisch auf Leukocytenfreiheit geprüft.

\section{Proteolytische Fermente}

1. Kristallines Trypsin (Novo), 24,2 Anson-Trypsineinheiten/g; 2. Kristalline Bakterienproteinase (Novo), entsprechend 22,2 Anson-Trypsineinheiten/g;

3. Kristallines Pepsin (Serva), kristalline Suspension, diisopropylfluorophosphat-behandelt, d. h. frei von Chymotrypsin, Trypsin und Aminosäuren.

Alle Fermentversuche wurden mit kristalliner Substanz angesetzt, um zusätzliche Serumverdünnungen zu vermeiden, da

$\left.{ }^{2}\right)$ Die Sedimente wurden freundlicherweise von den Behringwerken, Marburg, zur Verfügung gestellt. bereits bei Zugabe der Fermentinhibitoren zum Teil beträchtliche Serumverdünnungen in Kauf genommen werden mußten.

Fermentinhibitoren

Trasylol (Bayer), ist ein polyvalenter Inhibitor von Trypsin, Chymotrypsin, Kalliktein und Plasmin. Die Trypsinhemmkapazität zählt zu den höchsten bisher gefundenen. Zusammenfassung der Literatur bei Vogel, Trautschold und Werle (34). Pepsin wird durch Trasylol weder gehemmt noch wird Trasylol durch Pepsin angegriffen (35). Kathepsin C, Carboxypeptidase und die meisten Bakterienproteinasen werden durch Trasylol nicht gehemmt $(36,37)$. Nach Barnhart (38) soll Trasylol dagegen Kathepsin D und andere leukocytäre, lysosomale Proteasen hemmen. $1 \mathrm{mg}$ Trypsin wird durch 1250 Kallikrein-InaktivatorEinheiten (KIE) Trasylol inaktiviert. Verwendet wurden Ampullen zu je $5 \mathrm{ml}$, entsprechend $25000 \mathrm{KIE}$.

Diisopropjlfluorphosphat (Schuchatdt), ein hochtoxisches, öliges, organisches Phosphat, ist ein polyvalenter Esterasenhemmer. Diisopropylfluorphosphat hemmt Cholinesterase, Trypsin, Chymotrypsin und Kallikrein $(39,40)$, nicht aber Kathepsin B und C (19), Kathepsin D (41) und tryptische Carboxypeptidase (42). Es hemmt Trypsin komplett bei $0,4 \mathrm{~mm}$. $\mathrm{Da}$ die Angaben in der Literatur über die zur Hemmung erforderlichen Konzentrationen erheblich schwanken, andererseits die zellulären Enzymaktivitäten nicht bekannt waren, wurde willkürlich eine $0,1 \mathrm{M}$ Diisopropylfluorophosphat-Lösung hergestellt und den Reagenzien in steigenden Mengen zugesetzt.

Saponin, als 0,1proz. Lösung, soll nach Mounter und ATrYeH (24) eine völlige Hemmwirkung gegenüber Pepsin besitzen.

Phenylmethylsulfonylfluorid (Serva), von FAHRNEY und GoLD (43) als Ersatz für das sehr toxische Diisopropylfluorophosphat empfohlen, hemmt gleichfalls Trypsin und Chymotrypsin. Es war für diese Versuchstreihen jedoch unbrauchbar, da es sich nur in Isopropanol lösen ließ (Eiweißdenaturierung).

\section{Immunoelektrophorese}

Die Immunoelektrophorese wurde unter Verwendung des Barbital-Calciumlactatpuffers nach HrRsChFeld $(44,45)$ entsprechend einer früher mitgeteilten Modifikation (46) der von BAIrsch und Mitarbeitern (47) angegebenen Variante der immunoelektrophoretischen Mikromethode auf Glasplatten vorgenommen. Die $115 \times 300 \mathrm{~mm}$ messenden Spiegelglasplatten als Gelträger lassen in einem Untersuchungsgang maximal 80 Einzelbestimmungen zu. Als Immunseren dienten Anti-Gc-Seren vom Pferd und von der Ziege sowie ein monospezifisches Anti-Gc-Serum vom Kaninchen.

\section{Beschreibung der Versuche}

Vollblut und Citratblut der $3 \mathrm{Gc}$-Typen wurden zur Freisetzung der Zellproteasen mehrfach bei $-20^{\circ}$ eingefroren und aufgetaut. Diese Proben und nichtgefrorene Kontrollen wurden $1-4$ Stdn. bei $37^{\circ}$ inkubiert und anschließend immunoelektrophoretisch untersucht.

Frische Serumproben der 3 Gc-Typen wurden mit abgestuften Mengen homogenisierter Leuko- und Thrombocyten sowie leukocytenfreier Erythrocyten versetzt, und zwat jeweils $0,2 \mathrm{ml}$ Serum mit $0,001,0,01$ und $0,1 \mathrm{ml}$ unverdünnter LeukocytenThrombocytenaufschwemmung und jeweils $0,5 \mathrm{ml}$ Serum mit $0,5 \mathrm{~m} l$ Erythrocytenhämolysat. Alle Proben wurden mehrfach eingefroren und anschließend bei $37^{\circ}$ inkubiert. Die der Immunoelektrophorese vorausgehende Inkubation betrug 3-4 Stdn. bei den mit Leukocyten-Thrombocyten, und $24 \mathrm{Stdn}$. bis 11 Tage bei den mit Erythrocyten versetzten Seren. Auch hier wurden jeweils Kontrollen mit unzerstörten Blutzellen mitgeführt.

Seren der 3 Gc-Typen wurden mit kristallinem Pepsin, Trypsin, Bakterienprotease und Carboxypeptidase fermentiert, und zwar:

a) jeweils $1,0 \mathrm{ml}$ Serum mit $1,0,2,0$ und $3,0 \mathrm{mg}$ Pepsin; Inkubationszeit: 4 Stdn. bei $37^{\circ}$;

b) jeweils $0,5 \mathrm{~m} /$ Serum mit 0,5 und $1,0 \mathrm{mg}$ Trypsin; Inkubationszeit: 4 Stdn.; 
c) jeweils $1,0 \mathrm{ml}$ Serum mit 1,0 mg Bakterienprotease; Inkubationszcit: 25 Min. und 45 Min.;

d) jeweils $1,0 \mathrm{ml}$ Serum mit 10,0 $\mathrm{mg}$ Carboxypeptidase; Inkubationszeit: 4 Stdn.

Mit Ausnahme der Kontrollen wurden allen Proben die oben crwähnten Inhibitoren in einem Parallelversuch vor der Fermentaktivierung im Verhältnis $1: 1$ bis $1: 4$ zugesetzt, da die Aktivität

\section{Ergebnisse}

Aus den immunoelektrophoretischen Gc-Bildern ergab sich:

Trypsin, Bakterienprotease und die Eigenfermente aus den Blutzellen bewirkten eindeutige Gc-Transformationen. Abbildung 1 zeigt die Fermenteinwirkung des Trypsins $(0,5 \mathrm{mg} / 0,5 \mathrm{~m} /$ Serum) nach $4 \mathrm{stdg}$. Inkubation. Bei allen 3 Gc-Typen beginnt sich ein mit dem Gc-Protein identisches , $\alpha_{1}$-Globulin" horizontal abzuspalten. Die Pfeile markieren den Beginn der Aufspaltung. Das $\alpha_{2}$-Makroglobulin fängt an, sich anodenwärts zu verschieben. Die Buchstaben A, C und $\mathrm{E}$ bezeichnen jeweils die GcDefekttypen 1-1, 2-1 und 2-2, die Buchstaben B, D und $\mathrm{F}$ die entsprechenden Kontrollen.

Verdoppelt man die Trypsindosis, so wird die Transformation noch deutlicher (Abb. 2). Der 1-1-Typ weist jetzt ein klar erkennbares „, $\alpha_{1}$-Globulin“ auf; der Gc 2-1-Typ ist offensichtlich in 3 hintereinander liegende Fragmente gespalten, und $\operatorname{der}$ Gc 2-2-Bogen hat sich gestreckt und ist deutlich verlängert, so daß ein dem Typ 2-1 ähnliches Bild resultiert.

Die Einwirkung der Bakterienprotease $(1,0 \mathrm{mg} / 1,0 \mathrm{ml}$ Serum) bei 25 Min. Inkubation zeigt, daß der 1-1-Typ mit dem $\alpha_{2}$-Makroglobulin anodisch gewandert ist und sich zur Kathode und Anode hin in 3 Fragmente aufzusplittern beginnt (Abb. 3). Für den Gc-Typ 2-1 gilt das gleiche. Hier erkennt man gut die Linie des sich abspaltenden „, $\alpha_{1}$-Globulins“. Die Gc 2-2-Linie ist über dem deutlich anodisch gewanderten $\alpha_{2}$-Makroglobulin

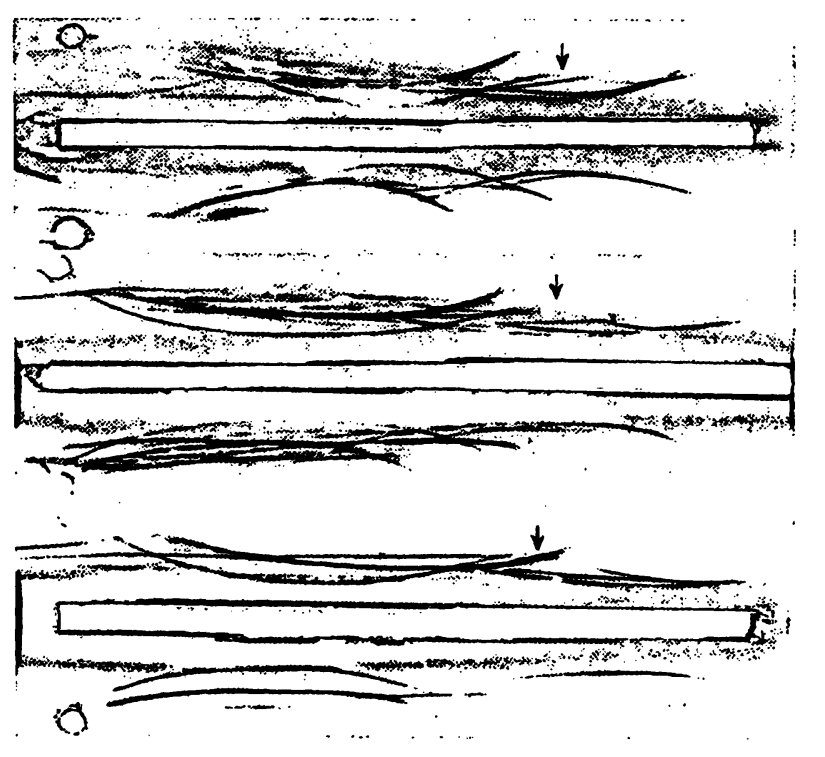

Abb. 1

Transformation der 3 trypsinbehandelten Typen Gc 1-1 (A), Oc 21 (C) und Gc 2-2 (E) nach 4stíndiger Inkubation. Entsprechende Kontrollseren bei $B, D$ und $F$. Die Pfeile kennzeichinen das sich $a b-$

B der Zellfermente eine unbekannte Größe darstellt.

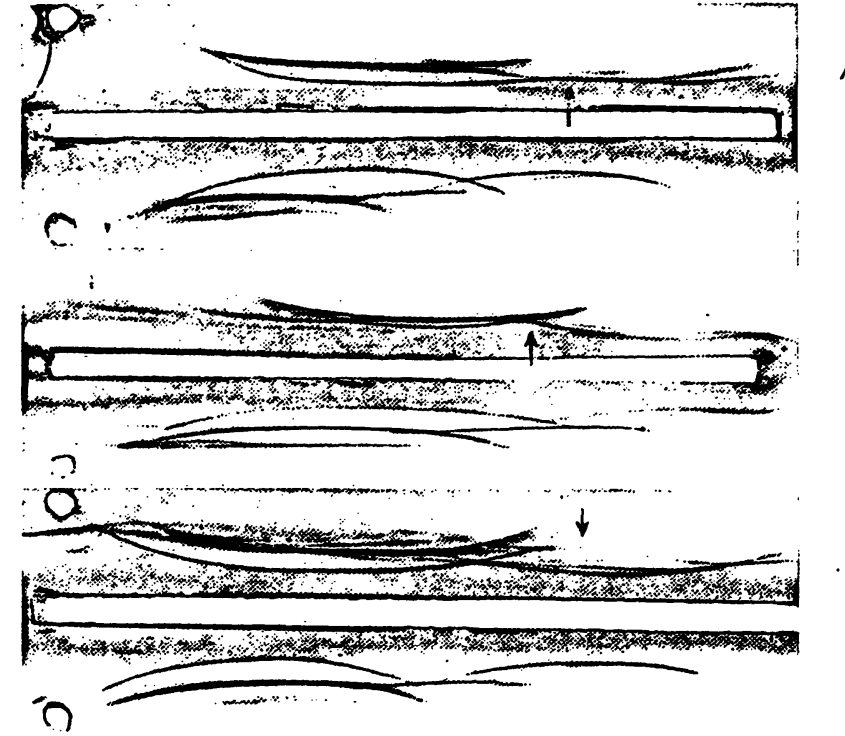

$A$

Abb. 2

Nach doppelter Trypsindosis deutliche Ausbildung des $\alpha_{1}$-Globulins bei Gc 1-1 (A) und Aufspaltung des Typs 2-1 in 3 Fragmente (C)

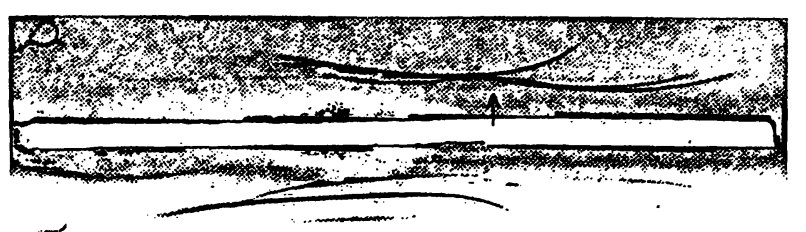

A

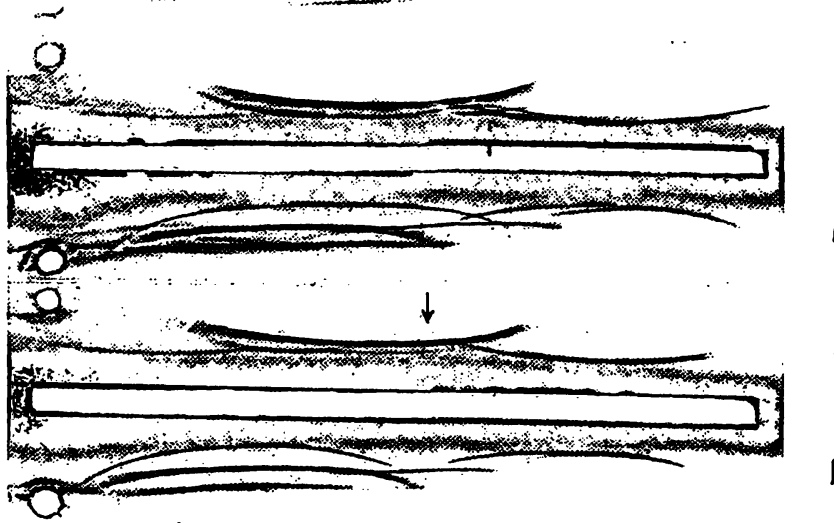

Abb. 3

A Fermenteinwirkung der Bakterienprotease nach 25 Min. Inkubation. Übergang in ein $\alpha_{1}$-Globulin ist hier beim Typ 2-1 besonders deutlich (C)

nahezu völlig in einen kathodalen und einen anodalen Anteil getrennt!

C Nach längerer Inkubationszeit mit der Bakterienprotease (45 Min.) stellt sich das „, $\alpha_{1}$-Globulin“ des 1-1-Typs deutlicher dar (Abb.4); im übrigen imponiert der 1-1-Typ als lang ausgezogene Linie zwischen Start und Albumin. Gc 2-1 und 2-2 zeigen eine fortgeschrittene Aufsplitterung, und alle 3 Typen erscheinen zur Kathode hin abgeschwächt.

Die Einwirkung der Leukocytenfermente $(0,001 \mathrm{ml}$ Leukocytenaufschwemmung/0,2 ml Serum) nach 4 stdg.
Inkubation läßt erkennen, daß der 1-1-Typ zuerst der Transformation unterliegt, dann der Gc 2-1-Typ und schließlich der Typ 2-2 (Abb. 5).

Die 10 fache Thrombocyten-Leukocytenextraktdosis bewirkt nach gleicher Inkubationszeit eine weitgehende 


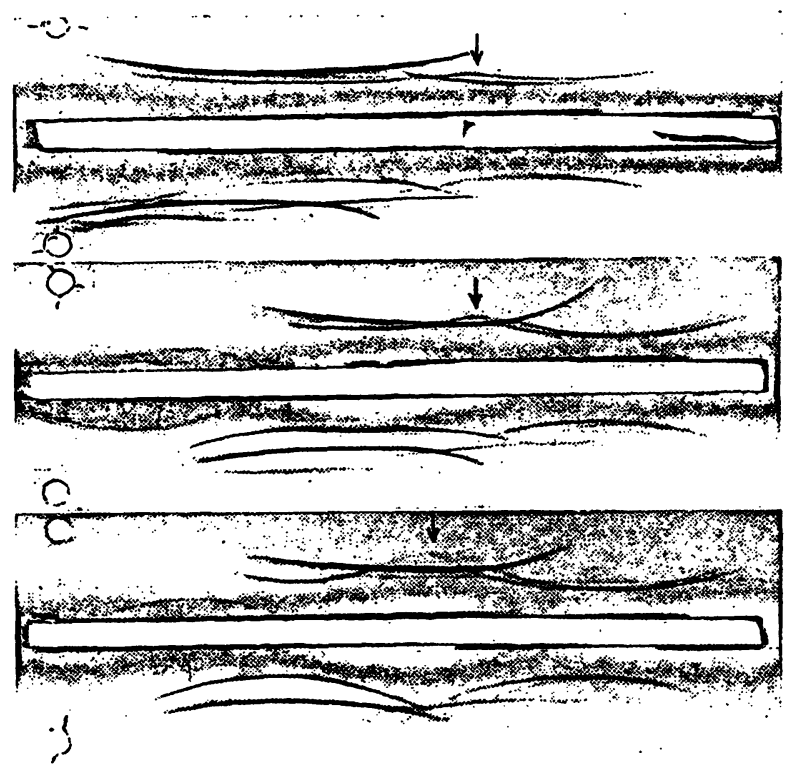

Abb. 4

Versuch wie unter Abb. 3 nach 45 Min. Inkubation. Fortgeschrittene Aufsplitterung und kathodenwärts Abschwächung der Gc-Linien

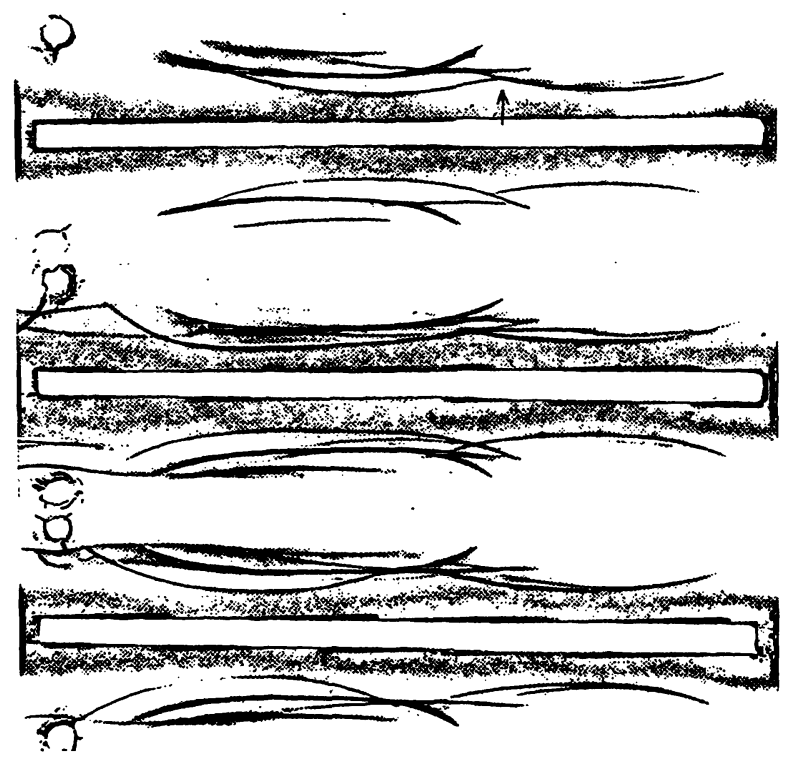

Abb. 5

Einwirkung der Leuko- und Thrombocytenfermente nach 4 Stdn. Inkubation. Bei Gc 1-1 ist bereits die Transformation in ein $\alpha_{1}$-Globulin zu erkennen

Umwandlung aller 3 Typen. Bei oberflächlicher Betrachtung imponiert der Gc-Typ 1-1 wie ein 2-1, der Typ 2-1 wie ein 2-2 und der Typ 2-2 wie ein 2-1 (Abb. 6). Bei weiterer Erhöhung der Extraktdosis um den Faktor 10 bietet sich das gleiche Bild wie nach Gefrierhämolyse am frischen Vollblut: alle 3 Typen gehen in ein , $\alpha_{1-}$ Globulin" über. Eine Diagnose ist nicht mehr möglich (Abb. 7).

Offensichtlich haben auch die Erythrocyten, wenn auch in weitaus geringerem Maße, proteolytisch wirksame Enzymaktivitäten. Nach 24 Stdn. Inkubation leukocytenfreien Erythrocytenhämolysates mit frischen Seren bietet sich folgendes Bild: Der Gc 1-1-Typ zeigt eine Verdoppelung des Bogens, das zugehörige $\alpha_{2}$ -

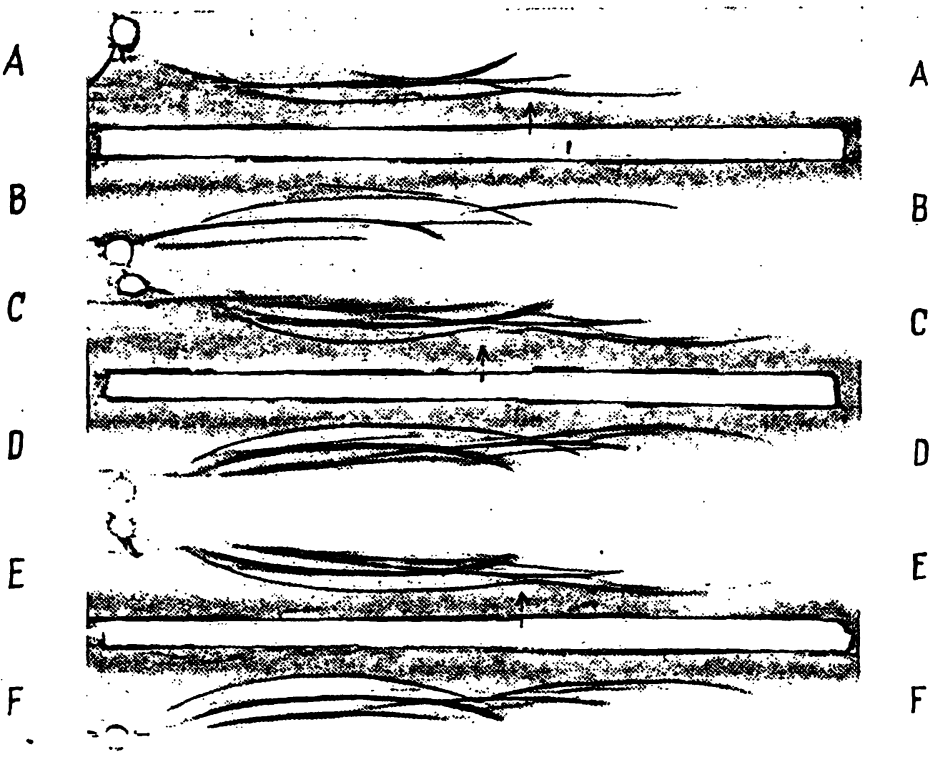

Abb. 6

Versuch wie unter Abb. 5 nach 10 facher Menge Leuko- und Thrombocytensediment. Stadium der Transformation, das zu Fehldiagnosen führt. Gc 1-1 kann fälschlich als 21 (A), Gc 2-1 als 2-2 (C) und $G$ 2-2 als 2-1 (E) abgelesen werden

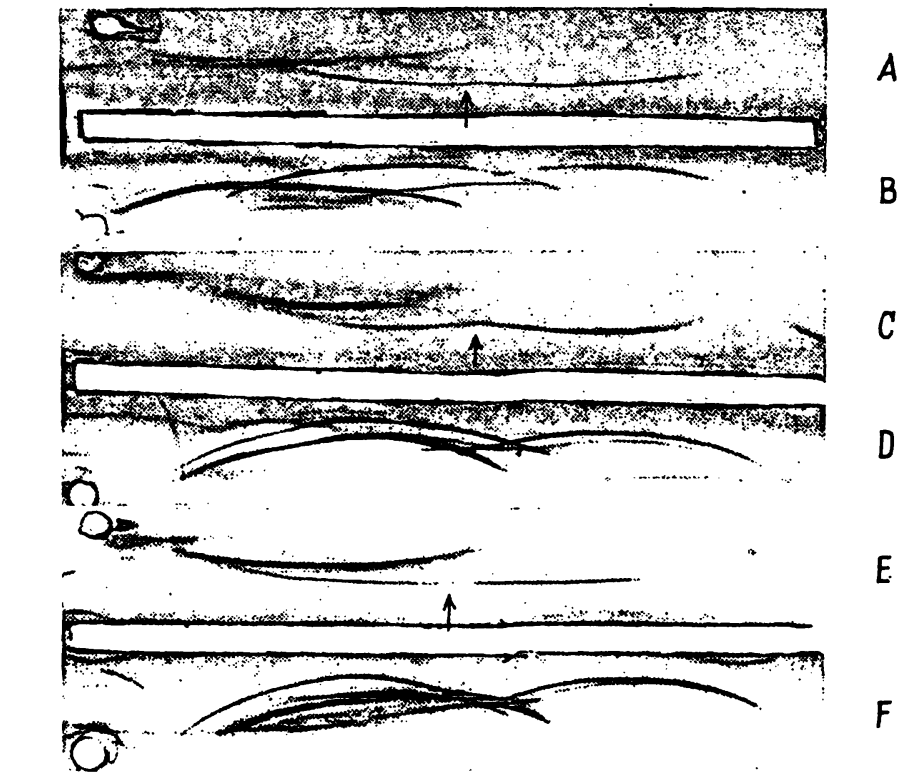

Abb. 7

Versuch wie unter Abb. 5 nach 100 facher Menge Leuko- und Thrombocytensediment. Vollständige Transformation aller Gc-Typen in ein $\alpha_{1}$-Globulin. Das gleiche Bild entsteht nach Gefrierhämolyse am Vollblut oder Citratblut

Makroglobulin eine deutlich verminderte elektrophoretische Beweglichkeit. Die Diagnostik des Gc-Typs 2-1 bereitet durch seine gegenläufige Verschiebung zum $\alpha_{2}$-Makroglobulin jetzt schon Schwierigkeiten (Abb. 8). Nach 4 Tagen Inkubation ist nunmehr das zum Gc 2-1 zugehörige $\alpha_{2}$-Makroglobulin eindeutig geringer gewandert, so daß der Gc 2-1-Typ, der übrigens ganz zart angedeutet ein „, $\alpha_{1}$-Globulin" erkennen läßt, als Gc 1-1 diagnostiziert werden könnte. Am wenigsten beeinflußt scheint der Typ 2-2 zu sein. Es fällt aber auf, daß gerade hier ein weiterer „Bezugsbogen“, das $\alpha_{1}$-Glykoprotein, nicht mehr an typischer Stelle, isondern - infolge verringerter Wanderungsgeschwindigkeit - zum $\alpha_{2}$-Globulinbereich hin verschoben, erkennbar ist (Abb. 9). 


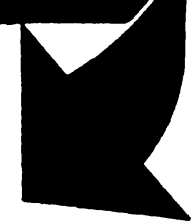

elektrisch beheizt, regelbar von $30-330^{\circ} \mathrm{C}$, für thermische Aufschlüsse, u. a. zur Stickstoffbestimmung biol. Substanzen, mikroanalyt. Untersuchungen in Laboratorien der

\section{Biochemie Mikrobiologie Klinische Chemie}

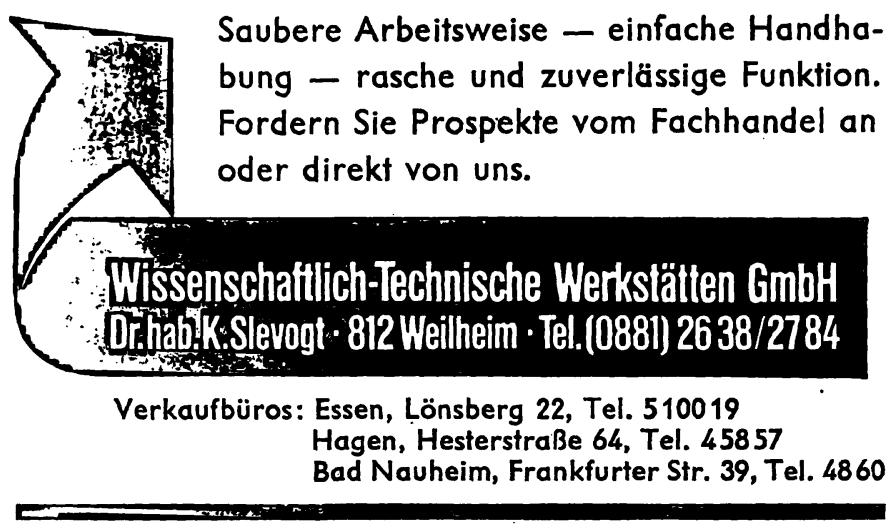

\section{ARBEITSMETHODEN}

DER MODERNEN NATURWISSENSCHAFTEN

Herausgegeben von Prof. Dr. KURT FrschBECK

\section{Dorfner}

\section{Ionenaustauscher}

Eigenschaften und Anwendungen

Von Dr. phil. Konrad Dorfner Diplom-Chemiker

2., neubearbeitete und erweiterte Auflage Mit 58 Abbildungen und 27 Tabellen.

Oktav. VIII, 208 Seiten. 1964. Plastikeinband DM 28,-

Die knappe Form der Darstellung wie auch die übersichtliche Gliederung und Beifügung von ausgewählten wesentlichen Tabellen und Abbildungen ermöglicht die rasche Information über Ionenaustauscher und ihre Anwendung. Dabei erweisen sich die als Beispiele für die Anwendung dargestellten Arbeitsvorschriften als wertvolle Ergänzung.

Bericbte über die gesamte Biologie

\section{Walter de Gruyter \& Co · Berlin}

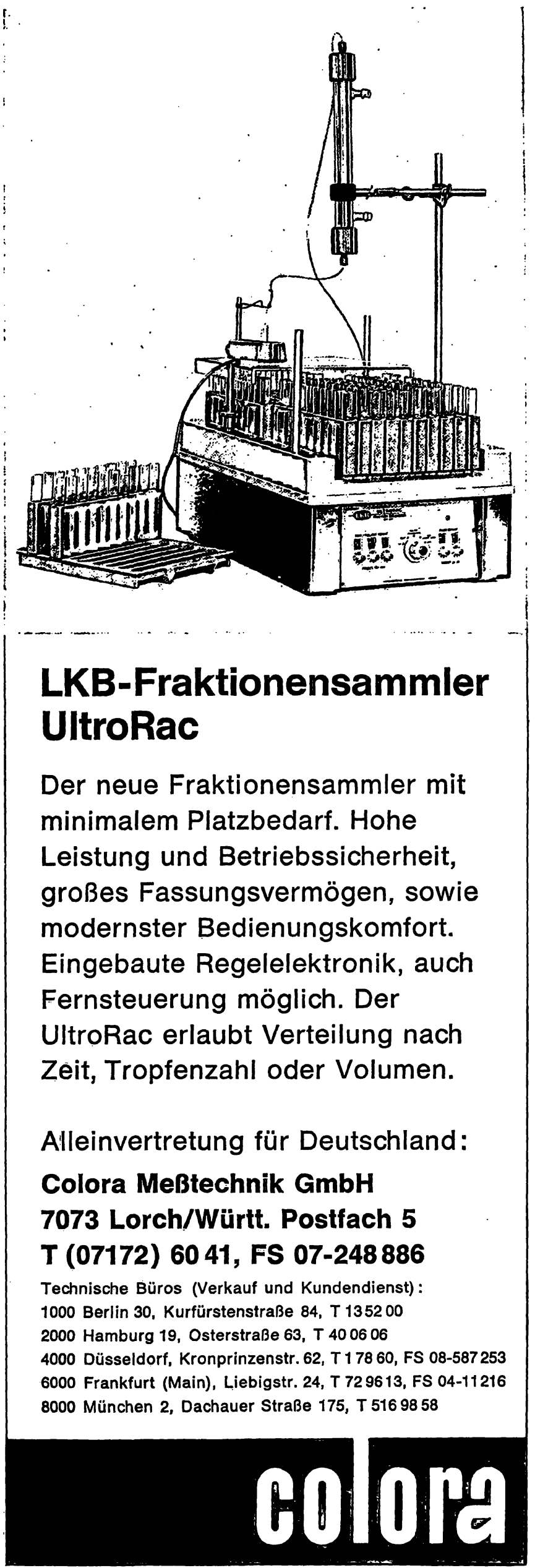




\section{AQUA PRO INJECTIONE}

Wir liefern Destillierautomaten mit Leistungen bis $500 \mathrm{l} / \mathrm{h}$. Die hervorragenden Eigenschaften des Spezialglases DURAN $50^{(B)}$ und die ausgereifte Konstruktion der Anlagen garantieren Wasserqualitäten, die den Forderungen der internationalen Pharmakopöen entsprechen. Deshalb werden vorzugsweise unsere Anlagen in der pharmazeutischen Industrie und in Krankenhäusern sowie Blutspendezentralen eingesetzt . . .

\section{... z. B. in der Blutspendezentrale} Bad Kreuznach

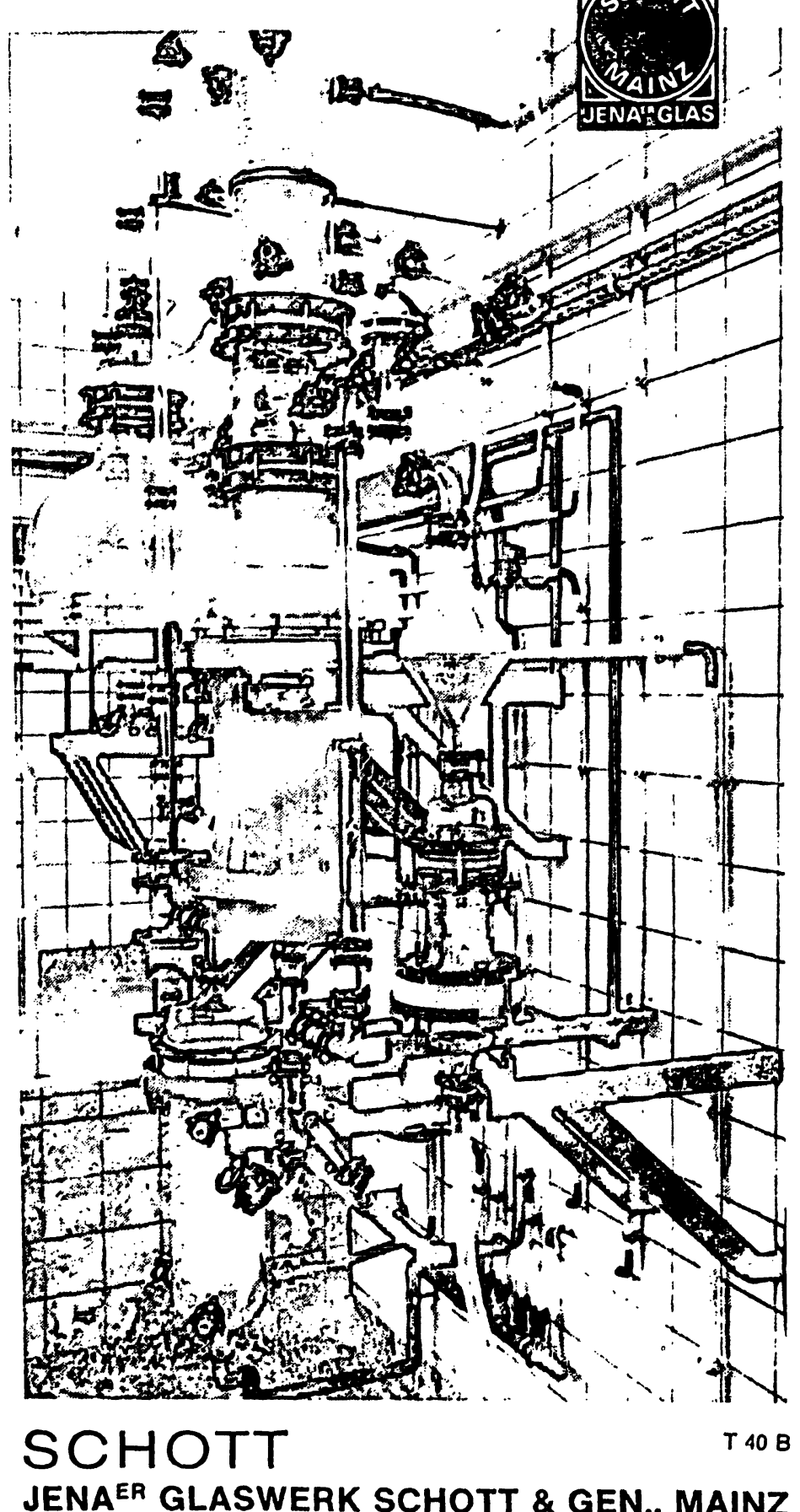

\section{Magenoperation und}

\section{Magenoperierter}

Prof. Dr. med. Heinrich Bartelheimer

Direktor der I. Medivinischen Universitäts-Klinik Hamburg

Prof. Dr. med. Hans-Joachim Maurer

Institut und Klinik für Medizinische Strahlenkunde der Universitât Düsseldorf

Prof. Dr. med. Hans W. Schreiber

Chefarzt der Chirurgischen Abteilung des Maricn-Krankenhauses. Hamburg

unter Mitwirkung von

Priv.-Doz. Dr. med. Kurt Müller-Wieland Oberarzt der I. Mcdizinischen Universitäts-Klinik Hamburg

Groß-Oktav. XVI, 489 Seiten. Mit 210 Abbildungen und 2 mehrfarbigen Tafeln. 1969. Ganzleinen DM 88,-

Durch die Zusammenarbeit verschiedener Disziplinen und durch dic Entwicklung neuer Methoden sind in der Gastrologic neuc Erkenntnisse über Form und Funktion des gesunden und ksanken Magens gewonnen worden. Die ebirurgiscben Leistungen wurden durch dic Einbezichung internistischer, röntgenologiscljer, miklearmediziniscber und vor allen Dingen biocbemiscber Verfalbren ergänzt. Gastroenterologen, Cbirurgen, Röntgenologen, Hämasologen, Osteologen und Paddiuter sowic l'ertreler anderer Fäcljer haben aus diesen Gründen hier dic Probleme der Magenoperation und der Magenopericrten dargestellt. Ërst langjährige und vergleichende Firfahrungen gestatten cine kritische Bewertung, wie sic jetzt hicr vorgelegt wird.

Das Buch wendet sich an alle Disziplinen der Medizin.

Verbindliche und cindeutige Aussagen zur Diagnostik und zum therapeutischen Vorgehen finden der praktizierende Arzl, der dic erste Indikation zu stcllen hat, ebenso wie Gastroenterologen, Internisten, Chirurgen und Röntgenologen. 


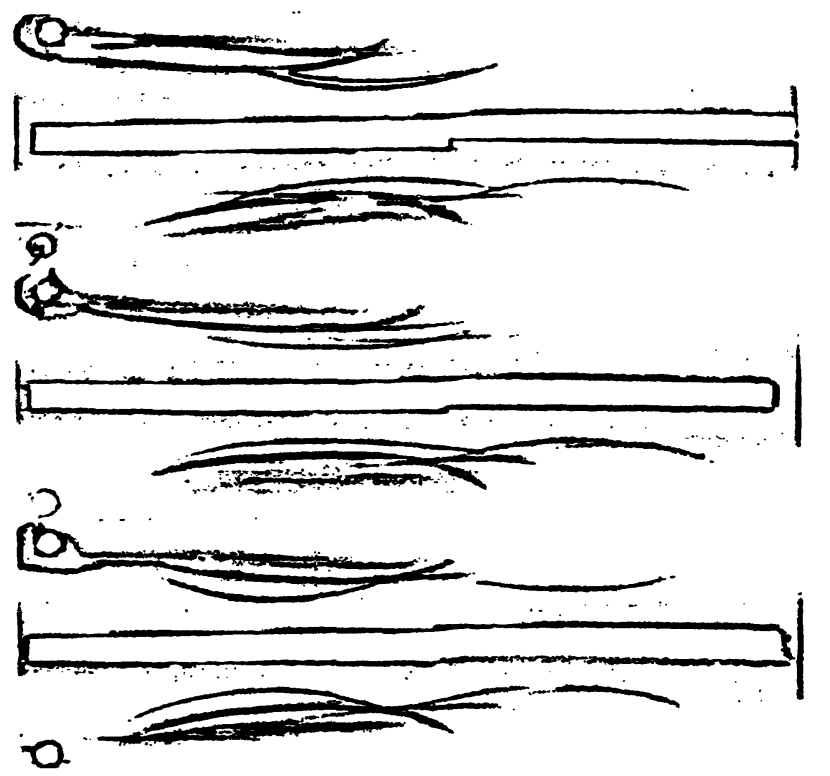

Abb. 8

Einwirkung der proteolytischen Erythrocytenfermente nach 24 stdg. Inkubation. Verdoppelung des Bogens bei Gc 1-1 und Verminderung der Laufgeschwindigkeit des zugehörigen $c_{2}$-Makroglobulins (A)

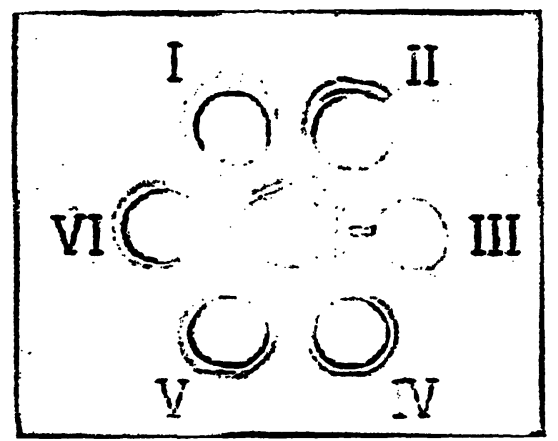

Abb. 10

Nachweis der immunologischen Identitāt der durch Proteolyse verursachten Fragmente des Gc-Globulins. Ouchterlony-Technik. Mitte: monospezifisches Anti-Gc-Serum vom Kaninchen; I Vollblut; II Serum; III Serum + Trypsin; IV Serum + Bakterienprotease; V Serum + Leuko- und Thrombocytenaufschwemmung; VI gefrierhämolysiertes Vollblut

Im Agargeldiffusionstest ließ sich beweisen, da $\beta$ sogar nach schwersten proteolytischen Verānderungen der Gc-Typen ihre immunologische Identitāt erhalten blieb. Abbildung 10 gibt die eindeutig koaleszierenden, wenn auch zum Teil etwas abgeschwächten Präzipitatlinien wieder. Die Sechserrosette war folgendermaßen beimpft: in der Zentralhöhlung monospezifisches Anti-Gc-Serum, in Höhlung I frisches Vollblut, in II frisches Serum, in III mit Trypsin fermentiertes Serum, in IV mit Bakterienproteinase fermentiertes Serum, in V Serum und Leukocyten-Thrombocytenaufschwemmung, in VI gefrierhāmolysiertes Vollblut.

Ohne fermentative Einwirkung waren selbst im Überschuß zugesetztes kristallines Pepsin sowie die Carboxypeptidase, die definitionsgemāß nur Polypeptide zu spalten vermag.

Mit Ausnahme des Trypsins, das sich durch Trasylol vollstāndig, durch Diisopropylfuorophosphat weniger befriedigend hemmen ließ, wurde keines der zelleigenen und der Fremdfermente gehemmt.
A

B

C

D

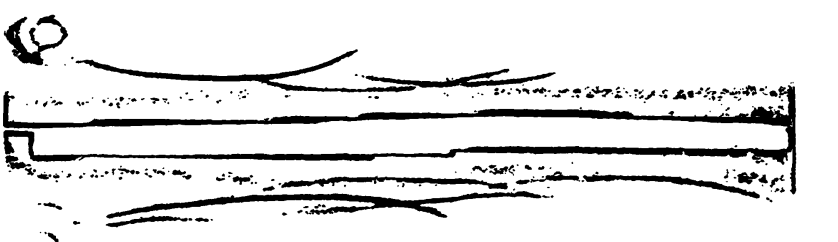

E

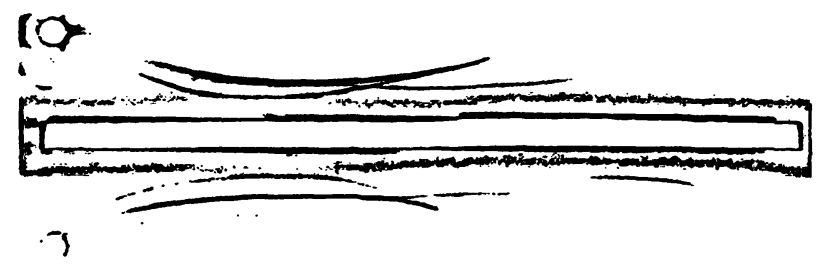

E

Abb. 9

Versuch wie unter Abb. 8 nach 4 Tagen Inkubation. Transformation des Gc 2-1 in ein $\alpha_{1}$-Globulin, das auBerdem wie ein Gc 1-1 imponiert (C)

Die mitgeführten Kontrollen an Vollblut-, Citratblutund Serumproben sowie an den mit Aufschwemmungen unzerstörter Leukocyten und Erythrocyten versetzten Seren waren negativ.

\section{Diskussion}

Die Transformation der Proteine ist abhängig von der Konzentration des Fermentes, von der Zeitdauer seiner Einwirkung und rom Temperaturoptimum. Insofern war das Ausmaß der Proteinschãdigung experimentell steuerbar. Nicht zu beeinflussen war hingegen die Transformation des Gc-Proteins am gefrierhāmolysierten Vollblut. So genügte bereits das $1-2$ malige Einfrieren und Auftauen mit anschließender Inkubation von 1 Std., ja sogar das kurze Aufbewahren im Kühlschrank, um die gleichen Gc-Verānderungen zu provozieren wie etwa nach 4 stdg. Inkubation bei $37^{\circ}$. Es fällt ferner auf, daß das $\alpha_{2}$-Makroglobulin durch die zelleigenen Fermente stets wesentlich stārker in seiner Position und auch in seiner Konturenschärfe verāndert wurde als durch die zugesetrten proteolytischen Fermente. Diese Beobachtung stiutzt gleichfalls die Annahme, daB die Kathepsine in ihrer Proteinsubstratspezifitāt nicht mit den Verdauungsfermenten identisch und offenbar in der lebenden Zelle funktionslos sind und erst nach Absterben der Zelle wirksam werden.

Die durch Einwirkung von Erythrocytenfermenten hervorgerufenen Proteinverānderungen stehen im Gegensatz zu den Befunden von Nerstrom (6), der keine Gc-Veränderungen, allerdings nach nur 18stdg. Inkubationszeit, beobachtet hat. Sie bestātigen jedoch die Befunde von Haschen $(30,31)$ über die in den Erythrocyten vorhandenen proteolytischen Enzyme, deren Fermentaktivitāt naturgemāß wesentlich geringer ist als die der Leukocyten, wie es auch aus den Versuchen zu ersehen ist. Trotz wesentlich lāngerer Inkubationszeit wirden weitaus geringere Transformationen induziert. 
Daß cs sich hicr in der Tat um proteolytische Enzyme und nicht um cinc Vortäuschung dieser Enzyme durch Einwirkung baktericller Proteasen handelt, geht weiterhin aus der Versuchsanordnung hervor. Es ließ sich nämlich zcigen, daß dic unter möglichst sterilen Bedingungen nach 11-tägiger Inkubation aufgetretenen Proteinveränderungen mit den nach 4 Tagen Inkubation crziclten völlig identisch waren. Für die Praxis ergibt sich jedenfalls hicraus, daß man bercits bei mehr oder wenigcr starker Hämolyse und nicht erst nach Zerfall der Leukocyten und Thrombocyten mit Gc-Transformationen, dic zu Fehldiagnosen führen können, rechnen muß. Dies gilt besonders für Blutproben, die bei mehrtägigem 'Transport hochsommerlichen Temperaturen ausgesct\%t waren.

Dic Ergebnisse erklären zwangsläufig auch die Beobachtungen von Nerstrøm (6) über atypische Gc-Muster an Blutfleckenextrakten. Sic crklären auch dic Befunde von HikSCHFEJ.D und Mitarbeitern (48) über das Auftreten eines $\alpha_{1}$-Globulins in Nabelschnurbluten bei Morbus hacmolyticus neonatorum. In beiden Fällen muß cinc Freisctzung der intrazellulären proteolytischen Enzyme (Kathepsine) durch dic Hämolyse angenommen werden.

Dic durch bakterielle und leukocytäre Proteasen hervorgerufenen Proteinveränderungen haben nun eine gewisse Ähnlichkeit mit der von HenNig und Hoppe (49) beschricbenen Gc-Variante "Gc Z“. Der Verdacht eines Zusammenhanges dieser Veränderungen mit den GcVarianten drängt sich auf. Wenn man jedoch berücksichtigt, daß inzwischen hierüber drei weitere, jeweils durch Nachuntersuchungen bestätigte Beobachtungen vorlicgen $(50,51,63)$, wird man eine Vortäuschung dieser Varianten durch enzymatische Einflüsse ausschließen und die Annahme cines zusätzlichen Allels am Gc-Locus, $\mathrm{Gc}^{z}$, als bercchtigt ansehen können. Schließlich darf cincs nicht übersehen werden: wenn fermentativ induzicrte Gc-Transformationen eine Gc-Variante Gc Z vortäuschen könnten, müßten die zwangsläufig mit diesen Defekttypen auftretenden allgemeinen Proteinveränderungen diese Fehldeutung erkennen lassen; denn jede induzierte Gc-Transformation führt auch zu Veränderungen ciniger anderer Serumproteine. Bei Anwendung eines polyvalenten Antiserums lassen sich beispielswcise nach Transformation des Gc-Typs durch leukocytäre Fermente auch schwcre proteolytische Veränderungen des Albumins, des $\alpha_{1}$-Glyko- und Lipoproteins und des $\alpha_{2}$-Makroglobulins nachweisen (Abb. 11).

Dic Nichthemmbarkeit der zelleigenen Fermente beweist, daß mindestens in bezug auf die Proteinsubstrat-

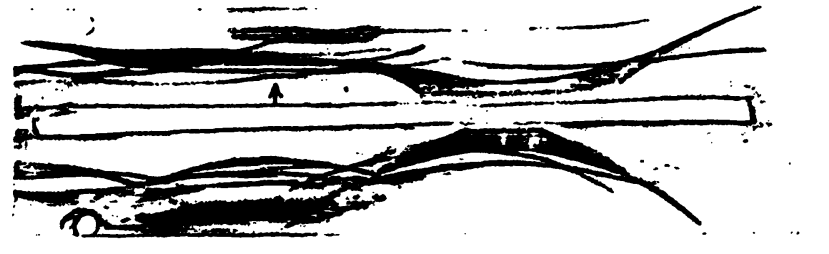

Abb. 11

Durch Leukocytenfermente induzierte Positionsänderungen und Transformationen des Albumins, $\alpha_{1}$-Glykoproteins, $\alpha_{2}$-Lipoproteins und der Gc-Linie 1-1 (A, Pfeil). Anti-Humanserum vom Pferd

spezifität kein Zusammenhang zwischen den Eigenfermenten der Blutzellen und den Verdauungsfermenten besteht. Dies war im Grunde schon deshalb zu vermuten, da offenbar die Antiproteasen des menschlichen Serums, $\alpha_{1}$-Antitrypsin (52), $\alpha_{1}$-Antichymotrypsin (53), Inter- $\alpha$-Trypsininhibitor (54), $\alpha_{2}$-Antiplasmin oder $\alpha_{2}$ Makroglobulin (55), mit hoher Inhibitorkapazität gegen Trypsin, Chymotrypsin und Plasmin ebenfalls nicht die zellulären Kathepsine hemmen können.

Trypsin oder trypsinähnliche Aktivitäten können also für die Gc-Transformationen nicht verantwortlich gemacht werden. Auch Pepsin oder pepsinähnliche Proteasen können für die Gc-Transformation nicht ursächlich sein. Die Unwirksamkeit des experimentell im Uberschuß zugesetzten kristallinen Pepsins ist erklärlich, da sein Wirkungsoptimum bei $\mathrm{pH}$-Werten von 1,5 bis 2,5 liegt und es bei Werten über 6 unstabil wird. Der $\mathrm{pH}-$ Wert des Blutes ändert sich nicht wesentlich nach Gefrierhämolyse und liegt nach eigenen Messungen selbst bei gefaultem 2 Jahre alten Blut noch zwischen $\mathrm{pH} 7$ und 8. Offenbar wird dieser Wert durch die Alkalireserve des Blutes sowie die Pufferwirkung der Plasmaeiweißkörper als Ampholyte auch außerhalb des Organismus sehr lange aufrechterhalten.

Abschließend ist festzustellen, $\mathrm{da} ß$ nach Kontamination des Serums mit Bakterien oder mit zellulären Proteasen jeder der 3 Gc-Typen zu Fehlbestimmungen Veranlassung geben kann. Von Prokop (56) vorgeschlagene Versuche, durch fermentative Behandlung der menschlichen Seren zusätzliche, möglicherweise genetisch determinierte, latent vorhandene Polymorphismen im Gc-System darzustellen, wie es beispielsweise bei den Transferrinen $(57,58,59,60)$ und einem $\alpha_{1}$ - und $\beta$-Globulin beschrieben worden ist $(61,62)$, erscheinen daher wenig erfolgversprechend. Eine Fermentierung erschwert die Gc-Klassifizierung unnötigerweise oder führt sogar zu völlig unbrauchbaren Resultaten.

Den Firmen Bayer (Leverkusen) und Novo Industrie (Mainz) sei an dieser Stclle für die Überlassung von Präparaten und Ferĩuenten gedankt.

\section{Literatur}

1. Sciuvirzz, H. E. und G. Schivick, Behringwerk-Mitt. $\mathcal{S}, 11$ (1957). - 2. Leirthorp, H. und 1. Leirnorf, Dtsch. Z. gerichtl. Mcd. 54, 286 (1963). - 3. Heirer, U. und M. Bolkenius, Dtsch. Z. gerichtl. Med. 58, 76 (1966). - 4. Forster, B. und H. JoAcinm, Dtsch. Z. gerichtl. Med. 63, 98 (1968). - 5. NerstrøM, B., Acta pathol. microbiol. Scand. 57, 495 (1963). - 6. NerstrøM, B., Acta pathol. microbiol. Scand. 60,540 (1964). - 7. Nerstrøm, B. und I. SKAfTE Jensen, Acta pathol. microbiol. Scand. 58, 257 (1963). - 8. Nerstrøm, B., B. Mansa und W. Frederiksen, Acta pathol. microbiol. Scand. 61, 474 (1964). - 9. STöss, B. und H. Petrenkofer, Zbl. Bakt. I Orig.; 190, 277 (1963). - 10. Jörgensen, G., Humangenetik 1, 303 (1965). - 11. Cleve, H. und A. Bearn, Progr. med. Genet. 2, 64 (1962). - 12. Cleve, H., Dic gruppenspezifischen Komponenten des menschlichen Serums. 
Hüthig-Verlag Heidelberg (1965). - 13. Reinskou, T., Vox Sang. 11, 70 (1966). - 14. Stein, U. und D. Platr, Klin. Wschr. 46, 1145 (1968). - 15. Coffey, J. und C. DE Duve, J. biol. Chemistry 243, 3255 (1968). - 16. Finkenstaedt, J. T., Proc. Soc. exper. Biol. Med. 95, 302 (1957). - 17. Barrett, A., Biochem. J. 104, 601 (1967). - 18. Bouma, J. und M. Gruber, Biochim. biophysica Acta Amsterdam 113, 350 (1966). - 19. FruroN, J. S., Cathepsins, in: Boyer, P., H. LARDY und K. MYrbäCK, The Enzymes, Bd. IV, 2. Aufl., S. 233 ff., Academic Press, New YorkLondon (1960). - 20. Pantlitscinko, M. und K. StatrmanN, Biochem. Z. 326, 252 (1955). - 21. Hascien, R. und K. KRUG, Z. ges. inn. Med. 20, 346 (1965). - 22. HASCHEN, R. und K. Krug, Nature (London) 209, 511 (1966). - 23. Frer, J., Cl. Borell, G. Horvath, B. Cullity und A. Vannotri, Blood 18, 317 (1961). - 24. Mounter, L. und W. ATIYeh, Blood 15, 52 (1960). - 25. Stiles, M. A. und J. Fraenkei-Conrat, Blood 32, 119 (1968). - 26. Rapoport, S., Medizinische Biochemie, 4. Auf., Volk und Gesundheit, Berlin (1966). - 27. BeESE, J., W. FARR, E. GrüNER und R. HASCHEN, Klin. Wschr. 44, 1049 (1966). 28. Haschen, R., Fol. haematol. 85, 288 (1966). - 29. Ohler, W., Klin. Wschr. 46, 737 (1968). - 30. HASCHEN, R., F. Groli, N. REHFELd und W. FARR, Acta biol. med. german. 13, 493 (1964). - 31. HASChEN, R., W. FARR und F. Groh, Fol. hacmatol. 83, 207 (1965). - 32. Morrison, W. und H. Neuratia, J. biol. Chemistry 200, 39 (1953). - 33. BETKE, K., Der menschliche rote Blutfarbstoff bei Fetus und reifem Organismus. Springer-Verlag, Berlin-Göttingen-Heidelberg (1954). - 34. VoGEL, R., I. TRAUTSCHOLd und E. Werle, Natürliche Proteinasen-Inhibitoren, G. Thieme-Verlag, Stuttgart (1966). - 35. Trautschold, I., E. WERLE und G. ZICKGRAF-Rüdel, Arzneimittelforsch. Aulendorf 16, 1507 (1966). - 36. Kraut, H. und N. Bhargava, HoppeSeyler's Z. physiol. Chem., 334, 236 (1963). - 37. KRAUT, H. und N. Bhargava, Hoppe-Seyler's $Z$. physiol. Chem. 348, 1498 (1967). - 38. Barnhart, M. J., C. Quitana, H. L. Lenon, G. B. BLUHM und I. M. RIDDLE, zit. nach HABERLAND und MATIS, Med. Welt 18, 1367 (1967). - 39. Jansen, E. F., M.-D. F. Nurting, R. Jang und A. K. Balls, J. biol. Chemistry 179, 189 (1949). - 40. Ooms, A. J. J., Nature (London) 190, 533 (1961). 41. Laskowski sr., M., B. Kassell, R. J. Peanasky und $M$.
Laskowskr jr., Endopeptidases, in: Hoppe-Scyler/Thierfelder (Hrsg.): Handbuch der physiologisch- und pathologisch-chemischen Analyse, Bd. VI/C, 10. Aufl., S. 229ff., Springer-Verlag, Berlin-Heidelberg-New York (1966). - 42. Hanson, H., Peptidasen (Exopeptidasen), in: Hoppe-Seyler/Thicrfelder (Hrsg.): Handbuch der physiologisch- und pathologisch-chemischen Analyse, Bd. VI/C, 10. Aufl. S. $1 \mathrm{f}$., Springer-Verlag, BerlinHeidelberg-New York (1966). - 43. Fahrney, D. E. und A. M. Gold, J. Amer. chem. Soc. 85, 997 (1963). - 44. HirsChFELd, J., Acta pathol. microbiol. Scand. 47, 160 (1959). - 45. Hirschifeld, J., Science Tools (LKB-Producter) 7, 18 (1960). - 46. HilgermanN, R., diese Z. 5, 317 (1967). - 47. Baitsch, H., I. KLose, K. Omoto und H. Ritrer, Ärztl. Lab. 10, 42 (1964). - 48. Hirschafeld, J., B. A. Nilson und B. NerstrøM, zit. nach Nerstrøm, Acta pathol. microbiol. Scand. 60, 540 (1964). 49. Hennig, W. und H. Hoppe, Vox Sang. 10, 214 (1965). 50. ClevVe, H., M. KRÜPE und A. ENSGRABER, Humangenctik 3 , 46 (1966). - 51. Luff, K. und G. Adebarr, Wiss. Z. Univ. Halle 17, 535 (1968). - 52. Schultze, H. E., K. Herde und H. Haupt, Klin. Wschr. 40, 427 (1962). - 53. Heimburger, N. und H. Hnupt, Clin. chim. Acta Amsterdam 12, 116 (1965). - 54. Heide, K., N. Heimburger und H. Haupt, Clin. chim. Acta Amsterdam 11, 82 (1965). - 55. Schultze, H. E., N. Heimburger, K. Heide, H. Haupt, K. Störiko und H. G. Schwick, Proc. 9th Congr. Europ. Soc. Haematol., Lisbon 1963, S. 1315, Karger, Basel-New York (1963). - 56. Prokop, O., Über ncuc Aspekte des ,immunochemical endgrouping" und über Serumpolymorphismen. Vortr. 47. Kongr. Ges. gerichtl. Med., Innsbruck 1968. - 57. PARKER, W. C. und A. G. BEARN, Science Washington 133, 1014 (1961). - 58. PARKER, W. C. und A. G. BeArn, J. exper. Med. 115, 83 (1962). - 59. Blumberg, B. S. und L. Warren, Biochim. biophysica Acta Amsterdam 50, 90 (1961). 60. Kaminski, M., Nature (London) 194, 27 (1962). - 61. VAN Hung, N., Acta biol. med. german. 21, 249 (1968). - 62. vaN Hung, N. und G. Geserick, Acta biol. med. german. 21, 679 (1968). - 63. Scheibe, E., E. Scheibe, E. Koch und G. Bundschur, in Dürwald, W. (Hrsg.), Aktuelle Fragen der gerichtlichen Medizin, Bd. III, S. 82, Wiss. Beitr. Univ. Halle (1968).
Dr. R. Hilgermann 355 Marburg Mannkopffstr. 2 